\title{
Ultrasound-guided vascular cannulation. Experience in critically-ill pediatric patients
}

\author{
José M. López Álvarez, M.D. ${ }^{a}$, Olivia Pérez Quevedo, M.D. ${ }^{a}$, Teresa Ramírez Lorenzo, M.D. ${ }^{b}$, \\ José M. Limiñana Cañal, M.D. ${ }^{b}$ and Juan F. Loro Ferrer, M.D. ${ }^{c}$
}

\begin{abstract}
Introduction. Central vascular cannulation in children is a highly complex technique and poses many difficulties. Vascular ultrasound can make this procedure easier.

Objective. To describe the characteristics of ultrasound-guided vascular cannulation in critically-ill pediatric patients.

Population and methods. Outcome measures prospectively recorded were vessels most frequently cannulated, their localization, the measurement of their diameter/depth, the success rate and complications developed, among others.

Results. One hundred and twenty four vascular punctures were performed in 86 pediatric patients. Vascular accesses were the femoral vein $(39.7 \%)$, followed by the femoral artery $(27.2 \%)$ and the internal jugular vein $(14.7 \%)$. Femoral vessels were localized at a depth of $0.75 \pm 0.25 \mathrm{~mm}$, with a mean diameter of $0.31 \pm 0.16 \mathrm{~mm}$. The depth of jugular vein vessels was smaller $(0.64 \pm 0.24 \mathrm{~mm})$ and their overall diameter, larger $(0.44 \pm 0.19 \mathrm{~mm})$. The mean number of attempts in ultrasound-guided cannulations was $2.2 \pm 1.3$. The success rate was $79 \%$ and was associated to a larger vessel diameter $(0.39 \pm 0.20 \mathrm{~mm}$ vs. $0.28 \pm 0.13 \mathrm{~mm}$, $p=0.01)$ and a lowernumber of attempts $(1.90 \pm 1.16$ vs. $3.45 \pm 1.77, p=0.001)$. Complications were accidental puncture of another vessel $(5.3 \%)$ and hematoma formation during puncture $(2.3 \%)$. Conclusions. In the pediatric patients studied, ultrasound-guided vascular cannulation allowed vessel visualization and measurement of their depth and diameter; the success rate was high and it was associated to a low complication rate. Key words: central venous catheterization, ultrasound, pediatrics.
\end{abstract}

http:/ / dx.doi.org/10.5546/aap.2018.eng.204

To cite: López Álvarez JM, Pérez Quevedo O, Ramírez Lorenzo T, et al. Ultrasound-guided vascular cannulation. Experience in critically-ill pediatric patients. Arch Argent Pediatr 2018;116(3):204-209.

\section{INTRODUCTION}

Central venous cannulation is a usual procedure for the management of critically-ill patients.

In pediatric patients, the technical complexity and difficulties are higher due to the following factors: a) greater difficulty to localize vessels based on anatomical landmarks; b) smaller vascular diameter which renders vascular palpation and visualization more difficult; c) greater capillary fragility which favors vein rupture; d) higher risk of puncturing or damaging non-desired structures; e) anatomical variation of vessels; f) clinical situations which require multiple and long-lasting vascular accesses (large preterm infants, chronic pediatric patients, long length of stay at the Intensive Care Unit), and g) less collaboration from patients. ${ }^{1-4}$

In pediatric patients, the failure rate associated to the technique based on anatomical landmarks ranges from $20 \%$ to $55 \%$, and the complications rate, from $10 \%$ to $25 \% .^{4-8}$ These complications, as well as vessel cannulation failure, increase in shock, dehydration, swelling or coagulopathy situations, which are generally associated to the criticallyill patient.

Vascular ultrasound is used in adult patients to facilitate ultrasoundguided vascular cannulation. In pediatric patients, it is being implemented gradually, reducing the failure rate to $1.2 \%-6 \%$, and the number of cannulation attempts, and has an associated decrease of complications between $5 \%$ and $6.5 \% .^{6-9}$

The purpose of this study was to describe the characteristics of ultrasound-guided vascular cannulation in critically-ill pediatric patients. 


\section{POPULATION AND METHODS}

Descriptive, prospective, observational study conducted along 4 years (January 2013 to December 2016) at a Pediatric Intensive Care Unit (PICU) with 10 multipurpose beds and a mean of 300 admissions per year, which included pediatric patients between 1 day old and 16 years old, who required an ultrasound-guided central venous cannulation as per the following protocol.

Ultrasound-guided localization and measurement of vessels to be cannulated in pediatric patients were performed, mainly the femoral artery (FA), femoral vein (FV) and internal jugular vein (IJV), since these were the most frequently cannulated vessels in pediatric patients.

The ultrasound cross-sectional (axial) plane with an out-of-plane approach was the most used view. Vascular cannulation was performed using the standard or modified Seldinger technique for peripherally-inserted central catheters. ${ }^{10}$

\section{DEFINITIONS}

- Vascular depth (Dp): Distance from the surface of the skin to the anterior wall of the vessel, measured by vascular ultrasound.

- Vascular diameter (Dm): Distance from the anterior wall to the posterior wall of the vessel, measured by vascular ultrasound.

- Axial (transverse) view with an out-of-plane approach: The ultrasound probe was placed perpendicularly to the vascular structure, thus forming an axis of approximately $90^{\circ}$. The visualization of vascular structures was circular.

- Success rate: Successful vascular cannulations among all the patients undergoing an ultrasound-guided vascular puncture.

- Success rate at first attempt: Percentage of correct vascular cannulations after only one attempt.

- Cannulation to relocate the guide: Puncture of the vessel in which blood spontaneously reflows or does so after aspiration with the syringe, and the insertion of the guide is achieved after moving the needle or modifying its angle.

- Puncture without cannulation: Puncture of the vessel in which blood spontaneously reflows or does so after aspiration with the syringe, without being able to insert the guide even after moving the needle or modifying its angle.

- Number of attempts: Number of punctures performed removing all the needle and introducing it again for correct vascular cannulation or needle visualization. The modification of the needle direction or depth for vascular cannulation or optimization of ultrasound visualization was not considered an attempt.

- Accidental puncture of another vessel: Puncture of a non-desired vessel.

- Hematoma: Decrease of the caliber of the vessel to be punctured or worse vessel differentiation regarding the infiltration into surrounding tissue due to blood extravasation after failed vascular puncture.

- Body surface area (BSA): Expressed in square meters $\left(\mathrm{m}^{2}\right)$ and calculated based on Mosteller formula. ${ }^{11}$

BSA: $\sqrt{\text { height }(\mathrm{cm}) \times \text { weight }(\mathrm{kg}) / 3600}$

The statistical analysis was performed using the Statistical Package for Social Sciences, version 19, for Windows (SPSS Inc., Chicago, IL, USA).

Qualitative outcome measures were summarized using their frequencies and percentages, and numerical outcome measures by their means, typical deviations and medians, which were analyzed using the Chi-Square test of independence and the Student's $t$ test for two independent samples, respectively. The level of significance was $5 \%$.

Children's parents or legal representatives were requested to sign an informed consent. The Study Protocol was conducted in accordance with the basic principles of the Declaration of Helsinki and the World Medical Association ${ }^{12}$, and was approved by the Hospital's Institutional Review Board.

The authors state there are no conflicts of interest to be declared in relation to this study.

\section{RESULTS}

Ninety eight pediatric patients were included in the study throughout 4 years. Out of these, 12 patients were excluded: 2 because their families did not give their Informed Consent; 6 because of the poor quality of their ultrasound images (patients with fluid overload, swelling or obesity), and 4 due to the lack of technical availability of the ultrasound machine. Finally, 86 patients were included in the study; they were subjected to 124 ultrasound-guided vascular punctures $(68 \%$ of veins, $32 \%$ of arteries). The annual percent increased from $5 \%$ to $42 \%$. The anthropometric characteristics of the pediatric population studied are presented in Table 1.

Vein vascular accesses most frequently used 
were the femoral access (58\%) followed by the jugular one $(23 \%)$, while the femoral artery was the most used $(86 \%)$ in arterial access.

Table 2 and Figure 1 show the depth (Dp) and diameter $(\mathrm{Dm})$ measurements of the main pediatric vessels studied. Femoral vessels were located at a higher Dp than jugular vessels: $0.75 \pm 0.25 \mathrm{~mm}$ vs. $0.64 \pm 0.24 \mathrm{~mm}, p=0.08$; and their mean Dm was smaller than that of the jugular vessels: $0.31 \pm 0.16$ versus $0.44 \pm 0.19 \mathrm{~mm}, p=0.08$.

The mean number of attempts for ultrasoundguided vascular cannulations was $2.23 \pm 1.34$.

The success rate for ultrasound-guided vascular cannulations was $79.4 \%$, ranging from $85 \%$ for jugular vessels to $78 \%$ for femoral vessels (83\% for veins and $70 \%$ for arteries). Figure 2 shows the success rate associated to the cannulated vessels. Other less frequently

TABLE 1. Characteristics of the pediatric population studied. $N: 86$

\begin{tabular}{|c|c|c|c|c|c|}
\hline & Punctures & Mean & SD & $95 \%$ CI & Range \\
\hline Age (months) & 124 & 55.56 & 62.12 & [44.06-66.36] & $0.70 \quad 216.00$ \\
\hline Height $(\mathrm{cm})$ & 124 & 88.41 & 38.71 & [81.40-95.08] & 44.00170 .00 \\
\hline Weight $(\mathrm{kg})$ & 124 & 17.79 & 16.24 & [14.89-20.61] & $2.40 \quad 60.00$ \\
\hline $\begin{array}{l}\text { Body surface } \\
\text { area }\left(\mathrm{m}^{2}\right)\end{array}$ & 124 & 0.64 & 0.43 & {$[0.56-0.71]$} & $0.17 \quad 1.58$ \\
\hline
\end{tabular}

$\mathrm{N}$ : number of patients; SD: standard deviation;

CI: confidence interval. cannulated vessels (like the axillary vein or artery, the brachial vein or artery, or the radial artery) yielded success rates of $100 \%$, but the number of cases was low and, therefore, not representative.

The success rate of ultrasound-guided vascular cannulation was associated to the following factors: a) a larger vessel diameter, $0.39 \pm 0.20 \mathrm{~mm}$ vs. $0.28 \pm 0.13 \mathrm{~mm}, p=0.01$ [95\% confidence interval, 95\% CI; range: -0.17 to -0.04 ]; b) a smaller number of attempts, $1.90 \pm 1.16$ versus $3.45 \pm 1.27, p=0.001$ [95\% CI; range: 1.06 to 2.05]. The success rate at the first attempt was $53 \%$, and reached $90 \%$ if 3 attempts were needed.

Puncture without cannulation was performed in $20.5 \%$ of cases, and the guide had to be relocated for a correct vascular cannulation in $34.8 \%$ of the cases. This was associated to younger patients $(36.8 \pm 53.6$ months vs.
TABLE 2. Ultrasound measurements of the main studied vessels among the pediatric population

\begin{tabular}{lcccccc}
\hline & N & Mean & SD & $\mathbf{9 5 \%}$ CI & \multicolumn{2}{c}{ Range } \\
\hline Dp-FA & 37 & 0.75 & 0.26 & {$[0.68-0.88]$} & 0.30 & 1.80 \\
Dp-FV & 54 & 0.77 & 0.30 & {$[0.68-0.83]$} & 0.40 & 2.00 \\
Dp-IJV & 20 & 0.65 & 0.24 & {$[0.55-0.76]$} & 0.30 & 1.30 \\
Dm-FA & 37 & 0.36 & 0.22 & {$[0.31-0.44]$} & 0.15 & 0.82 \\
Dm-FV & 54 & 0.37 & 0.20 & {$[0.30-0.41]$} & 0.16 & 1.01 \\
Dm-IJV & 20 & 0.45 & 0.19 & {$[0.37-0.54]$} & 0.18 & 0.80 \\
\hline
\end{tabular}

$\mathrm{N}$ : number of vessels measured; Dp: depth; Dm: diameter; FV: femoral vein; FA: femoral artery; IJV: internal jugular vein; measures are expressed in cm; SD: standard deviation; CI: confidence interval.

FIGURE 1. Measurement of vascular depth and diameter in a 12-year-old girl (left) and a 23-month-old boy (right)
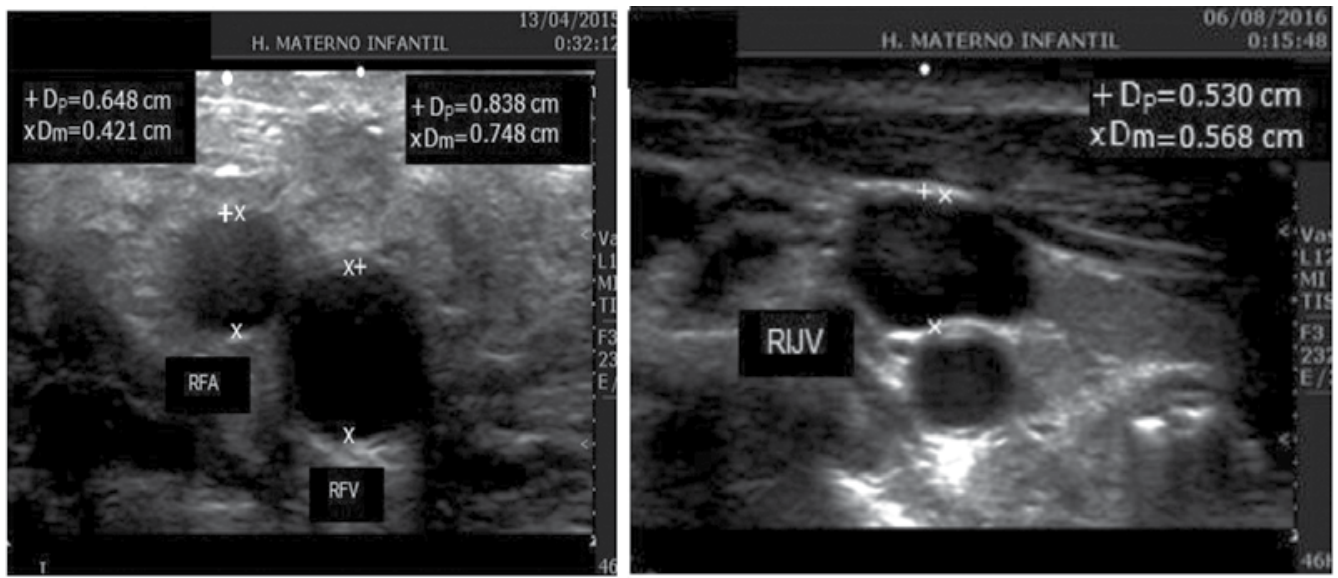

RFA: right femoral artery; RFV: right femoral vein; Dp: depth; Dm: diameter; RIJV: right internal jugular vein. 
$78.6 \pm 64.3$ months; $p=0.001$ [95\% CI; range: 19.81 to 66.54$]$ ) and smaller body surface area $\left(0.52 \pm 0.39 \mathrm{~m}^{2}\right.$ vs. $0.79 \pm 0.42, p=0.001[95 \% \mathrm{CI}$; range: 1.06 to 0.44$]$ ).

The following complications can be mentioned in relation with ultrasound-guided vascular cannulation in pediatric patients: accidental puncture of another vessel $(5,3 \%)$ and hematoma formation during puncture $(2.3 \%)$.

\section{DISCUSSION}

Through the use of ultrasound, this study allows to locate those vessels which are most frequently cannulated in pediatric patients, to measure them, to make their cannulation and relevant maneuvers easier, and to lower the complication rate related to the technique. Facing the difficulties of cannulating children, particularly younger children, ultrasound is an extremely useful tool.

Achieving a venous access in pediatrics may be a challenge for medical as well as nursing staff. This situation was clearly described by Orlowski in $1984,{ }^{13}$ who coined the following statement: "My kingdom for an intravenous line".

Even in vascular cannulations performed by experienced and qualified staff, intravenous access may be difficult to achieve in children, with failure rates between $20 \%$ and $55 \%$ and complication rates from $10 \%$ to $25 \%$, depending on the different series. ${ }^{4-8}$
In 2001, the Agency for Healthcare Research and Quality ${ }^{14}$ concluded that placing a central catheter with the help of ultrasound guidance was one of the most underused procedures, and that its incorporation to medical practice would improve the safety of patients.

There is a positive trend towards using the ultrasound-guided cannulation technique in critically-ill patients, which has been increasing throughout the last decade, ${ }^{15-18}$ and was also confirmed for pediatric patients. This fact was reflected in our work, with an increase of ultrasound-guided vascular cannulation use in the past 4 years.

Femoral and jugular vessels are the most frequently cannulated in pediatric patients. ${ }^{4}$ When analyzing their location and depth and diameter measures, it can be observed that femoral vessels are located deeper and have a smaller diameter than jugular vessels. ${ }^{18}$ These data might support the recommendation of IJV cannulation with the help of ultrasound, since most of the time, pediatric patients present the two most favorable conditions: a superficial location and a larger vascular diameter. ${ }^{19,20}$

Regarding vascular depth and diameter, it should be pointed out that ultrasound-guided vascular cannulation success rate falls below $56 \%$ in vessels with a diameter smaller than $0.3 \mathrm{~cm}$, and achieves a $92 \%$ success in vessels with a diameter larger than $0.6 \mathrm{~cm}$; hence, the likelihood of success for ultrasound-guided

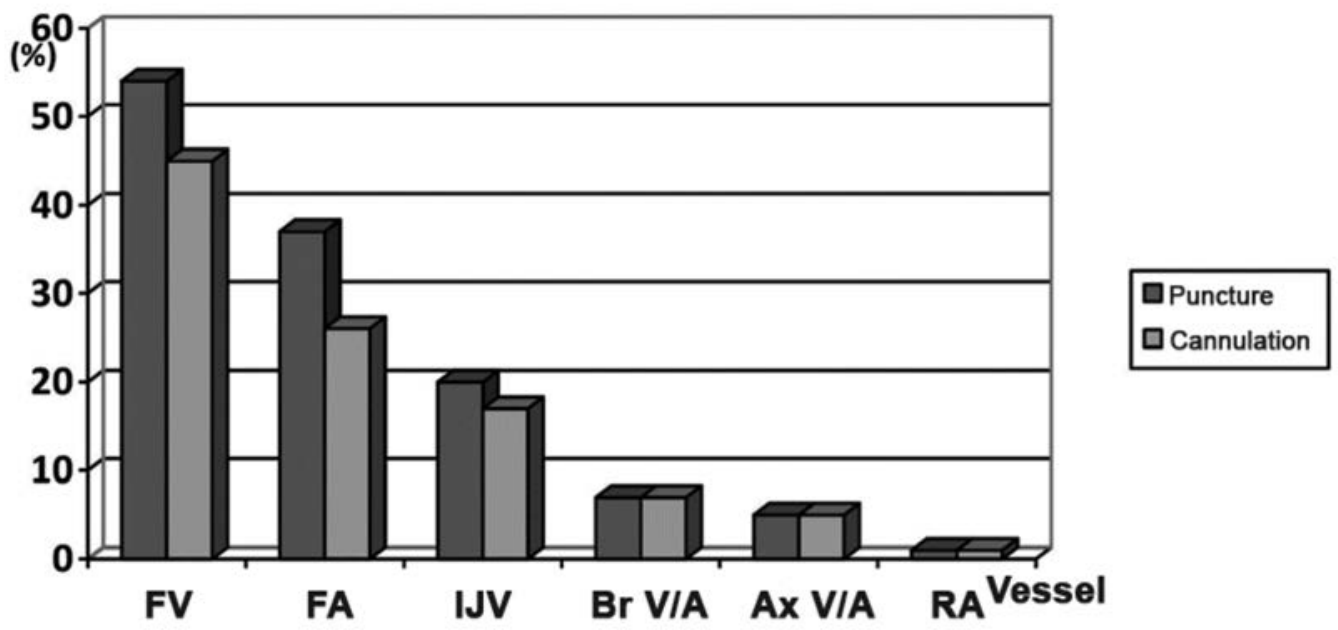

FV: femoral vein; FA: femoral artery; IJV: internal jugular vein; Br V/ A: brachial vein or artery; Ax V/A: axillary vein or artery; RA: radial artery. 
vascular cannulation increases by 1.79 per each millimeter increase in vascular diameter, and that beyond $1.6 \mathrm{~cm}$, vascular depth represents a decrease in the success rate. ${ }^{21}$ Furthermore, vascular cannulation can be optimized through the relocation maneuver of the guide or puncture needle. The best ultrasound approach is still under debate. ${ }^{21-24}$

The success rate recorded in our study is slightly lower than that of other published series. ${ }^{6-9}$

Yet, it is worth noting that a $90 \%$ success rate was achieved with a third attempt and that, in about $20 \%$ of the cases, it was possible to puncture the vessel but not to cannulate it. Within a population of 169 adult patients, Panebianco et al. ${ }^{21}$ observed an ultrasound-guided vascular cannulation success rate of $90 \%$ after 3 attempts in peripheral veins.

The complication rate was similar to that presented by other authors. ${ }^{6-9}$ Iwashima et al., ${ }^{25}$ mentioned that compared to a $32 \%$ for anatomical landmark, FA accidental puncture occurred in 7\% of pediatric patients when ultrasound was used. A study carried out by Suk et al., ${ }^{26}$ points out that this extremely high rate of arterial puncture may be due to the anatomical variations of vessels, which can achieve a femoral artery / vein overlapping as high as $74 \%$ in children. The same happens with cervical vessels, since the internal carotid artery can overlap the IJV or present these anatomical variations in relation to the internal carotid artery in as much as $54 \%$ of cases. ${ }^{27}$

In comparison with the standard technique which uses anatomical landmarks, the use of ultrasound to cannulate central vessels in children reduces the number of attempts resulting in a lower complication rate. ${ }^{28}$ Most complications are associated to the multiple attempts to puncture the vessel with the needle to achieve cannulation. ${ }^{14,27,29,30}$ The risk of complications significantly increases after two punctures of the same vessel, ${ }^{28}$ and this increase is six times higher after three attempts. ${ }^{30}$ Likewise, Ueda et al. ${ }^{31}$ conclude that a larger diameter is significantly associated to the success rate of a first attempt.

Despite the results obtained and the advantages described regarding the use of vascular ultrasound for the vascular cannulation of pediatric patients, this technique presents some limitations which should be noted: a) it requires a learning curve; b) it is limited as regards vascular depth and diameter; c) it becomes more challenging in younger patients; $d$ ) it requires either the pediatric patient cooperation or his / her previous sedation-analgesia to allow an optimum ultrasound visualization; and e) it is necessary to improve the development of equipment adapted to pediatric patients to achieve the ideal results in ultrasound-guided vascular cannulation.

This study underscores that ultrasound is a costeffective, harmless, low-complexity technique for critically-ill pediatric patients. These characteristics, together with the optimization of vessel visualization and the fact that it renders cannulation easier, make it a recommendable technique and encourage the development of a protocol for both vascular puncture and cannulation.

\section{CONCLUSIONS}

In the pediatric patients studied, ultrasoundguided vascular cannulation allows vessel visualization and measurement of their depth and diameter; it has a high success rate and is associated to a low complication rate.

\section{REFERENCES}

1. Rauch D, Dowd D, Eldridge D, et al. Peripheral difficult venous access in children. Clin Pediatr (Phila) 2009;48(9): 895-901.

2. Larsen P, Eldridge D, Brinkley J, et al. Pediatric peripheral intravenous access: does nursing experience and competence really make a difference? I Infus Nurs 2010;33(4):226-35.

3. Yen K, Riegert A, Gorelick MH. Derivation of the DIVA score: a clinical prediction rule for the identification of children with difficult intravenous access. Pediatr Emerg Care 2008;24(3):143-7.

4. Chiang VW, Baskin MN. Uses and complications of central venous catheters inserted in a pediatric emergency department. Pediatr Emerg Care 2000;16(4):230-2.

5. Froehlich CD, Rigby MR, Rosenberg ES, et al. Ultrasoundguided central venous catheter placement decreases complications and decreases placement attempts compared with the landmark technique in patients in a pediatric intensive care unit. Crit Care Med 2009;37(3):1090-6.

6. Alderson PJ, Burrows FA, Stemp LI, et al. Use of ultrasound to evaluate internal jugular vein anatomy and to facilitate central venous cannulation in paediatric patients. $\mathrm{Br} J$ Anaesth 1993;70(2):145-8.

7. Verghese ST, McGill WA, Patel RI, et al. Comparison of three techniques for internal jugular vein cannulation in infants. Paediatr Anaesth 2000;10(5):505-11.

8. Verghese ST, McGill WA, Patel RI, et al. Ultrasoundguided internal jugular venous cannulation in infants: a prospective comparison with the traditional palpation method. Anesthesiology 1999;91(1):71-7.

9. Bruzoni M,Slater BJ, Wall J, et al. A prospective randomized trial of ultrasound- vs landmark-guided central venous access in the pediatric population. J Am Coll Surg 2013;216(5):939-43.

10. Seldinger SI. Catheter replacement of the needle in percutaneous arteriography; a new technique. Acta Radiol 1953;39(5):368-76.

11. Mosteller RD. Simplified calculation of body-surface area. $N$ Engl J Med 1987;317(17):1098. 
12. World Medical Association. World Medical AssociationDec laration of Hels inki: ethic al principles for medical research involving human subjects. JAMA 2013;310(20):2191-4.

13. Orlowski JP. My kingdom for an intravenous line. Am J Dis Child 1984;138(9):803.

14. Pede S, Uguccioni M. [AHCPR/AHRQ guidelines. Agency for Health Care Policy and Research and Agency for Health Care Research and Quality]. Ital Heart J 2001;2 (Suppl 1): 60-8.

15. Leyvi G, Taylor DG, Reith E, et al. Utility of ultrasoundguided central venous cannulation in pediatric surgical patients: a clinical series. Paediatr Anaesth 2005;15(11):953-8.

16. Jaques PF, Mauro MA, Keefe B. US guidance for vascular access. Technical note. J Vasc Interv Radiol 1992;3(2):427-30.

17. LaRueGD. Efficacy of ultrasonography in peripheral venous cannulation. J Intraven Nurs 2000;23(1):29-34.

18. Pérez-Quevedo O, López-Álvarez JM, Limiñana-Cañal JM, et al. Design and application of model for training ultrasound-guided vascular cannulation in pediatric patients. Med Intensiva 2016;40(6):364-70.

19. Feller-Kopmal D. Ultrasound-Guided internal jugular access. Chest 2007;132(1): 302-9.

20. Turker G, Kaya F, Gurbet AI, et al. Internal jugular vein cannulation: an ultrasound-guided technique versus a landmark-guided technique. Clinics (Sao Paulo) 2009;64(10):989-92.

21. Panebianco NL, Fredette JM, Szyld D, et al. What you see (sonographically) is what you get: vein and patient characteristics associated with successful ultrasoundguided peripheral intravenous placement in patients with difficult access. Acad Emerg Med 2009;16(12):1298-303.

22. Hatfield A, Bodenham A. Portable ultrasound for difficult central venous access. Br J Anaesth 1999;82(6):822-6.
23. Vogel JA, Haukoos JS, Erickson CL, et al. Is long-axis view superior to short-axis view in ultrasound-guided central venous catheterization? Crit Care Med 2015;43(4):832-9.

24. Chittoodan S, Breen D, O'Donnell BD, et al Long versus short axis ultrasound guided approach for internal jugular vein cannulation: a prospective randomised controlled trial. Med Ultrason 2011;13(1):21-5.

25. Troianos CA, Kuwik RJ, Pasqual JR, et al. Internal jugular vein and carotid artery anatomic relation as determined by ultrasonography. Anesthesiology 1996;85(1):43-8.

26. Iwashima S, Ishikawa T, Ohzeki T. Ultrasound-guided versus landmark-guided femoral vein access in pediatric cardiac catheterization. Pediatr Cardiol 2008;29(2):339-42.

27. Suk EH, Lee K-Y, Kweon TD, et al. Ultrasonographic evaluation of the femoral vein in anaesthetised infants and young children. Anaesthesia 2010;65(9):895-8.

28. Duffy M, Sair M. Cannulation of central veins. Anaesth Intensive Care Med 2007;8(1):17-20.

29. Akl E, Kamath G, Yosuico V, et al. Thomboprophylaxiz for patients with cancer and central venous catheters: A sistematic review and a meta-analysis. Cancer 2008;112(11):2483-92.

30. Hernández Hernández MA, Álvarez Antoñan C, Pérez Ceballos MA. Complicaciones de la canalización de una vía venosa central. Rev Clin Esp 2006;206(1):50-3.

31. Eisen LA, Narasimhan M, Berger JS, et al. Mechanical complications of central venous catheters. J Intensive Care Med 2006;21(1):40-6.

32. Ueda K, Puangsuvan S, Hove MA, et al. Ultrasound visual image-guided vs Doppler auditory-assisted radial artery cannulation in infants and small children by non-expert anaesthesiologists: a randomized prospective study. $\mathrm{Br} J$ Anaesth 2013;110(2):281-6. 